\title{
Tips for learning and teaching evidence-based medicine: introduction to the series
}

\author{
Peter C. Wyer, Sheri Keitz, Rose Hatala, Robert Hayward, Alexandra Barratt, Victor Montori, \\ Eric Wooltorton, Gordon Guyatt
}

§ See related article page 353

M edical educators have embraced evidence-based medicine (EBM) since its introduction as an innovative approach to medical practice and education in the early 1990s ${ }^{1,2}$ The Royal College of Physicians and Surgeons of Canada, the College of Family Physicians of Canada and the US Accreditation Council of Graduate Medical Education include EBM skills among their mandated core competencies for residency programs. However, despite the availability of authoritative texts, ${ }^{3,4}$ teaching clinicians the basic EBM skills of formulating questions, finding the relevant literature efficiently, appraising that literature and applying it to their patient care remains challenging. ${ }^{5-7}$ The article by Alexandra Barratt and associates ${ }^{8}$ on page 353 of this issue of CMAJ constitutes the first in a new series aimed at facilitating this task.

It seems that nearly every general medical journal has its own series devoted to helping clinicians understand and apply the basic principles of EBM. What distinguishes this new CMAf series is that it targets not only clinician learners but also the teachers of EBM principles. These teachers regularly communicate the principles of critical appraisal to clinician learners in a variety of settings, including ward rounds, at the bedside, during journal club exercises and in formal lectures and seminars. We felt that we could offer some advice to help make the most of these teaching opportunities.

There are thus 2 versions of each article, one for learners of the EBM principle in question and one for their teachers. The learners' version will appear in print in $C M A 7$, and the related teachers' version will be published online only at www.cmaj.ca/cgi/content/full/171/4/353 /DC1 (which can be accessed as an online appendix to the learners' version). The online teachers' version will also give readers access to a variety of extra features, including interactive teaching exercises and other tools, such as PowerPoint slides. Registered subscribers to the Fournal of the American Medical Association or the AMA Archives journals will have access to an even broader set of tools, through links to the online collection of educational materials associated with the Users' Guides to the Medical Literature. ${ }^{4}$

In this introduction to the series, we outline the methods employed by the Evidence-Based Medicine Teaching
Tips Working Group to create the components of the package.

\section{How were the articles developed?}

The teachers' and learners' versions of the articles are the result of the ongoing Evidence-Based Medicine Teaching Tips project. The goal of this project is to assemble teaching approaches developed and used over a period of years by experienced EBM teachers and to put them into a form that will allow other educators to use them effectively. Most of the educators participating in the project teach regularly at an annual workshop called "How to Teach Evidence-Based Clinical Practice" at McMaster University. Many contributors to this series are also members of the Evidence-Based Medicine Working Group, which was responsible for developing the Users' Guides to the Medical Literature ${ }^{4}$ from which we have drawn the concepts and some of the visual approaches used in the tips series.

In collecting the tips, we first identified several target areas. Participants submitted brief descriptions of approaches that they had developed and used, including an outline of any examples employed. We then collated the descriptions and reviewed them together to decide how best to package them in manuscript form.

In developing the teachers' version of each manuscript, we were guided by Irby's concept of "teaching scripts" as an important tool of medical educators. Drawing upon a concept of "illness scripts" as a method of organizing clinical knowledge, ${ }^{9}$ Irby described the use of teaching scripts by outstanding clinical teachers in a traditional medical education model. ${ }^{10,11}$ These scripts, developed through repetitive teaching of specific content to similar groups of learners, contained the key teaching points for a topic. Irby considered teachers' identification of learners' typical errors and misconceptions regarding a topic to be a crucial element in the development of effective teaching scripts and hence of effective clinical teaching. ${ }^{11}$ Thus, the tips presented in this CMAJ series are constructed to respond to learners' common struggles with understanding the concepts central to EBM.

In the final phase of development of a particular manu- 
script, a member of the group who had not previously used the tip conducted a "field test." These field tests helped us to ensure that the descriptions of the teaching approaches were as clear as possible. Field tests also allowed us to identify and include variations in learner response to the concepts.

We have found that clinical learners respond best to presentations of EBM topics when they are offered as "easily digestible bites" or mini-lessons, presented in tandem with clinical problem-solving. ${ }^{12}$ Learners generally find graphic representations and tables more informative than formulas and equations. What characterizes the tips series is the "bite-size" framework and, in the teachers' versions, the attempt to describe the interactive process that unfolds when experienced EBM teachers use these tips. Teachers may apply these mini-lessons in a variety of settings where time is limited.

Ideally, educators would base their teaching strategies on approaches that have undergone rigorous prospective testing according to the same principles that we have come to expect of studies of the therapies we administer to our patients. Unfortunately, this level of scrutiny is rarely available for teaching methods. ${ }^{13}$ A number of obstacles, including lack of validated outcome measures, lie at the heart of this limitation. ${ }^{14,15}$ Formal empirical testing of our teaching tips is beyond the scope of the current project. Our own experience in using these approaches provides the evidence base supporting the suggestions.

\section{What's in store for readers}

The articles in this series cover a broad range of concepts, from estimating risk, estimating precision and understanding estimates of interrater reliability to the impact of heterogeneity on systematic reviews. We believe that clinicians will find the learners' versions useful as quick reference guides. EBM teachers will enjoy the scripted versions of the teaching tips and the online teaching tools that will be available to them.

We in turn are interested in learning what happens when clinical teachers use the tips and in discovering how the tips might be further improved or modified for use in different settings. Therefore, the online teachers' version of each tip includes a moderated discussion. Over time, we expect to incorporate new insights into the interactive versions of the tips.

From the Columbia University College of Physicians and Surgeons, New York, NY (Wyer); Durham Veterans Affairs Medical Center and Duke University Medical Center, Durham, NC (Keitz); the Department of Medicine, University of British Columbia, Vancouver, BC (Hatala); Centres for Health Evidence, University of Alberta, Edmonton, Alta. (Hayward); the School of Public Health, University of Sydney, Sydney, Australia (Barratt); Department of Medicine, Mayo Clinic College of Medicine, Rochester, Minn. (Montori); the Canadian Medical Association Fournal, Ottawa, Ont. (Wooltorton); the Departments of Medicine and of Clinical
Epidemiology and Biostatistics, McMaster University, Hamilton, Ont. (Guyatt); and the Evidence-Based Medicine Teaching Tips Working Group (Wyer, Keitz, Hatala, Hayward, Barratt, Montori, Guyatt)

Competing interests: None declared.

Contributors: Peter Wyer coordinated all phases of the Evidence-Based Medicine Teaching Tips project and of manuscript development and contributed to the development of the format of the teachers' and learners' versions of the articles in the series. Sheri Keitz designed the protocol for and conducted the field tests of the teachers' version of the manuscripts. Rose Hatala contributed to the conceptua design of the teachers' version of the manuscripts and to the protocol used by the authors of those articles; she also shared responsibility for quality review of the manuscripts once completed. Robert Hayward designed and edited the interactive online version of each article. As the first author of part 1 of this series, Alexandra Barratt contributed to the design and format of the teachers' and learners' versions of the manuscripts. As the first author of several other parts of the series, Victor Montori contributed to the design and format of the teachers' and learners' versions of the manuscripts. Eric Wooltorton contributed to the design and content of the learners' version of the manuscripts. Gordon Guyatt edited all manuscripts for accuracy and consistency of content and conception, advised on all aspects of project development and contributed a large proportion of the teaching approaches used in the manuscripts. All of the authors contributed to the writing and revision of this commentary.

\section{References}

1. Guyatt G. Evidence-based medicine [editorial]. ACP 7 Club 1991;114(2):A-16.

2. Evidence-Based Medicine Working Group. Evidence-based medicine. A new approach to teaching the practice of medicine. FAMA 1992;268:2420-5.

3. Sackett DL, Haynes RB, Guyatt GH, Tugwell P. Clinical epidemiology: a basic science for clinical medicine. 2nd ed. Boston: Little Brown and Co; 1991.

4. Guyatt G, Rennie D, editors. Users' guides to the medical literature: a manual for evidence-based clinical practice. Chicago: AMA Press; 2002.

5. Audet N, Gagnon R, Ladouceur R, Marcil M. L'enseignement de l'analyse critique des publications scientifiques médicales est-il efficace? Révision des études et de leur qualité méthodologique. CMA7 1993;148(6):945-52.

6. Norman GR, Shannon SI. Effectiveness of instruction in critical appraisal (evidence-based medicine) skills: a critical appraisal. CMA7 1998;158(2):177-81.

7. Coomarasmy A, Taylor R, Khan KS. A systematic review of postgraduate teaching in evidence-based medicine and critical appraisal. Med Teach 2003 25:77-81.

8. Barratt A, Wyer PC, Hatala R, McGinn T, Dans AL, Keitz S, et al, for the Evidence-Based Medicine Teaching Tips Working Group. Tips for learners of evidence-based medicine: 1 . Relative risk reduction, absolute risk reduction and number needed to treat. CMA7 2004;171(4):353-8.

9. Schmidt HG, Norman GR, Boshuizen HP. A cognitive perspective on medical expertise: theory and implication. Acad Med 1990;65:611-21.

10. Irby DM. How attending physicians make instructional decisions when conducting teaching rounds. Acad Med 1992;67:630-8.

11. Irby DM. What clinical teachers in medicine need to know. Acad Med 1994; 69:333-42.

12. Sackett DL, Straus SE, Richardson WS, Rosenberg W, Haynes RB. Evidencebased medicine: how to practice and teach EBM. 2nd ed. New York: Churchill Livingstone; 2000.

13. Parkes J, Hyde C, Deeks J, Milne R. Teaching critical appraisal skills in health care settings [Cochrane review]. In: The Cochrane Database of Systematic Reviews, Issue 2, 2004. Chichester, UK: John Wiley \& Sons.

14. Hatala R. Is evidence-based medicine a teachable skill? Ann Emerg Med 1999 34:226-8.

15. Hatala R, Guyatt G. Evaluating the teaching of evidence-based medicine. ZAMA 2002;288:1110-2.

Correspondence to: Dr. Peter C. Wyer, 446 Pelhamdale Ave. Pelham NY 10803 USA; fax 212 305-6792; pwyer@worldnet .att.net

The complete list of members of the Evidence-Based Medicine Teaching Tips Working Group appears with part 1 of the series, ${ }^{8}$ page 353 of this issue. 